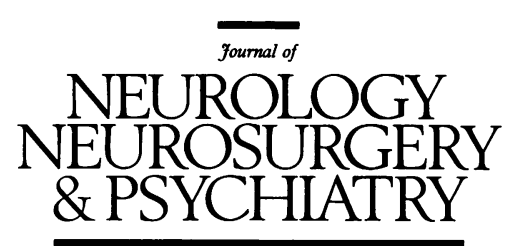

Editorial

\title{
Cellular pathology of multiple system atrophy: a review
}

Multiple system atrophy (MSA) has been an enigma both clinically and pathologically. Clinically, both the nomenclature and nosology of the disease have been surrounded by confusion and controversy. Similarly, the neuropathological definition reflected clinical uncertainties and was further hindered by non-specific histopathological manifestations of the disease. Neuronal degeneration and loss, accompanied by astrocytosis in various, often disparate parts of the CNS presented diagnostic and nosological problems. These histological abnormalities occur in a wide range of cerebral disorders, particularly in neurodegenerative diseases. Moreover, MSA, unlike other neurodegenerative disorders, lacked, until recently, any pathognomonic lesions. Most of these diseases have histological hallmarks, in addition to neuronal loss, which serve as diagnostic criteria. Plaques and tangles in Alzheimer's disease, Pick cells and Pick inclusions in Pick's disease, Lewy bodies in Parkinson's disease, and swollen neurons and corticobasal inclusions in corticobasal degeneration are important histological signposts pointing to the diagnosis. Yet until recently, no such pathognomonic features have been associated with MSA.

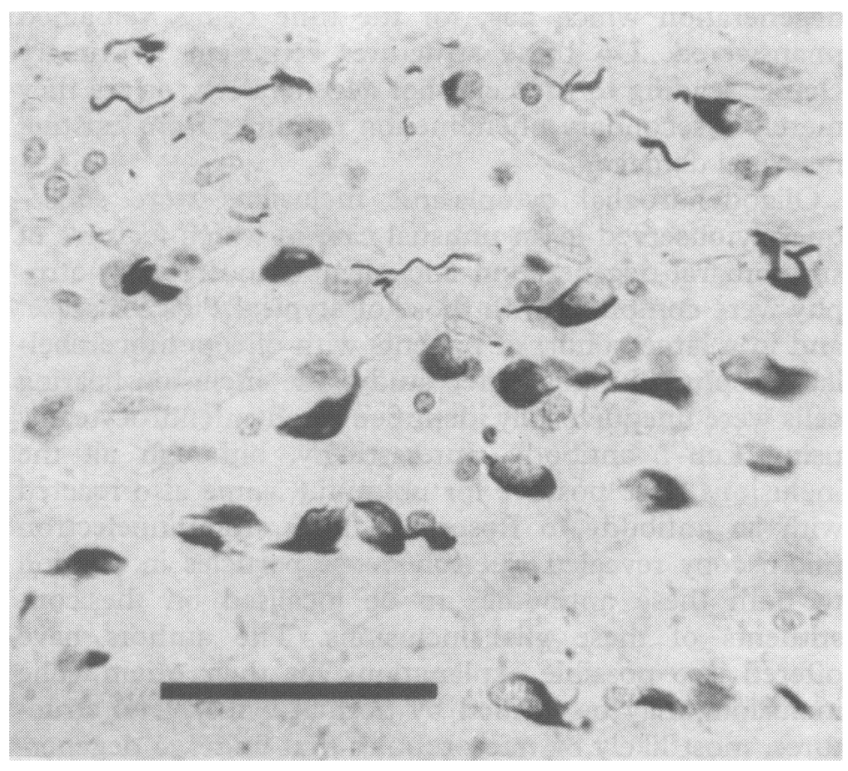

Figure 1 Argyrophilic glial cytoplasmic inclusions and abnormal cell processes (neuropil threads) in the basis pontis. Bar $=0.1 \mathrm{~mm}$. Gallyas silver impregnation.

\section{Glial cytoplasmic inclusions}

Recent developments in the cellular pathology of MSA have contributed considerably to the nosological definition of the disease and, at a more practical level, have increased the accuracy of neuropathological diagnosis. Various intracellular inclusions have been recently described in MSA and the involvement of the cortex has been also realised. The discovery of the glial cytoplasmic inclusions, however, was the single most important development. ${ }^{1}$

In 1989 Papp et al ${ }^{1}$ described glial cytoplasmic inclusions in the CNS of 11 patients with various combinations of striatonigral degeneration, olivopontocerebellar atrophy and Shy-Drager syndrome. These inclusions were first noted in silver impregnation developed by Gallyas $^{23}$ (fig 1) to demonstrate Alzheimer-type neurofibrillary changes and, although their configuration sometimes superficially resembled neurofibrillary tangles, their cellular location, measurements, ultrastructure, immunocytochemical profiles, and regional distributions were all different from these Alzheimer-type changes. Most glial cytoplasmic inclusions (GCIs) were localised in the white matter and appeared to be accompanied by an increase in the number of interfascicular oligodendroglial cells and pallor or loss of myelin staining. Histological, ultrastructural, and immunocytochemical findings have all indicated that these inclusions occur in oligodendrocytes.

In low-power electron micrographs, the main component of the GCIs appeared to be randomly arranged, loose filaments (fig 2), whereas higher magnification revealed tubular profiles, as suggested by their longitudinal view. In cross sections they were microtubular profiles consisting of a round or ovoid wall with a diameter of 20-30 nm between the two outer surfaces enclosing a clear centre. Immunocytochemically, the GCIs stained positively with antibodies to ubiquitin (figs 3 and 4), tau protein and to $\alpha$ - and $\beta$-tubulin. They gave negative reactions with antibodies to other cytoskeletal antigens, however, including actin, vimentin, desmin, cytokeratin, glial fibrillary acidic protein and neurofilament proteins. As GCIs were not found in age- and sex-matched controls who had other neurological diseases, the authors concluded that GCIs represent a cellular change characteristic of MSA and the three clinical syndromes are various manifestations of the same disease. ${ }^{1}$

The presence of these inclusions was soon confirmed by other groups. Nakazato et $a^{4}$ observed so-called oligodendroglial microtubular tangles in the brainstem, cerebellum and basal ganglia in association with severe 


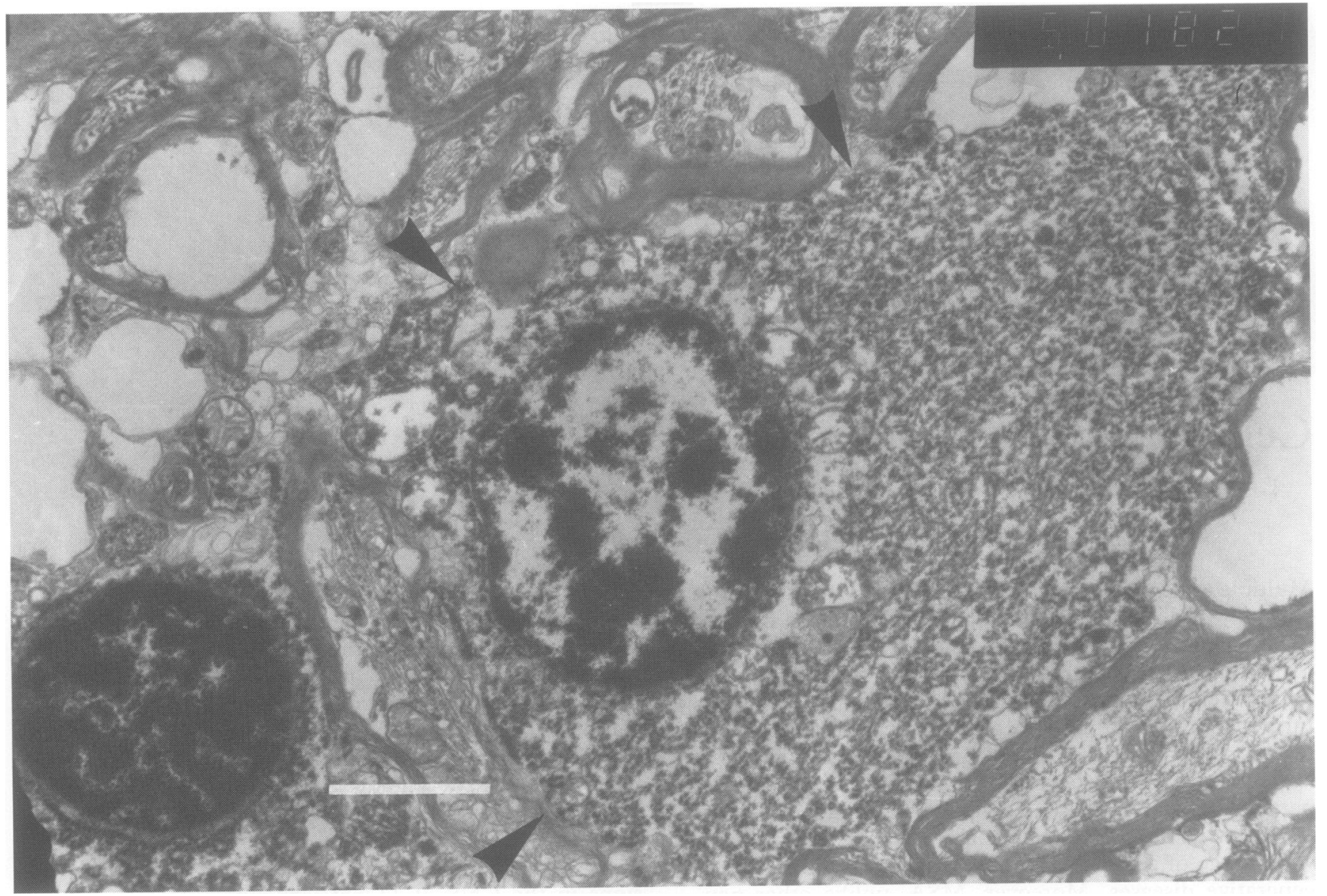

Figure 2 Low power electron micrograph of a glial cytoplasmic inclusion. Arrowheads indicate the border of the inclusion-bearing oligodendroglial cell which is surrounded by myelinated axons. Bar $=2 \cdot 5 \mu \mathrm{m}$.

neurodegenerative changes in the brains of patients with olivopontocerebellar atrophy. These oligodendroglial inclusions immunostained with antibodies to tubulin, but not with other antibodies, including one to tau protein. Immunocytochemistry for ubiquitin has not been carried out. Ultrastructurally, they confirmed the findings of Papp et $a l^{1}$ : the oligodendroglial inclusions were composed of altered microtubules with a diameter between 20-30 nm. In the same year, Kato and Nakamura described cytoplasmic argyrophilic inclusions in neurons of the pontine nuclei of patients with olivopontocerebel-

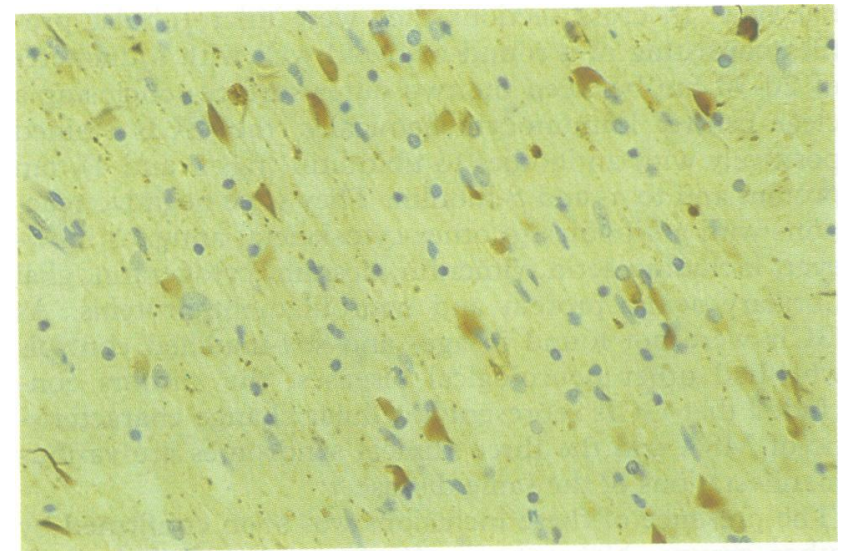

Figure 3 Immunocytochemistry for ubiquitin shows many positively stained inclusions in the pons. ABC method (Dako). Magnification $\times 125$, increased by $240 \%$ for publication. lar atrophy. They immunostained positively with an antibody to ubiquitin, but did not stain with any other cytoskeletal antibody. Ultrastructurally the inclusions were composed of fibrillary structures resembling microtubules with a diameter ranging from $24 \mathrm{~nm}$ to $40 \mathrm{~nm}$. Their outer surface was covered by osmiophilic granules and they were intermingled with a few filaments of $10 \mathrm{~nm}$ in diameter. The finding of these neuronal inclusions has raised a fundamental question about neurodegeneration which has, for the time being, remained unanswered. Do these structures represent a primary change leading to the neuronal degeneration, or are they merely a secondary phenomenon resulting from existing neuronal damage?

Oligodendroglial cytoplasmic inclusions were subsequently observed in an unusual case in which features of striatonigral degeneration and olivopontocerebellar atrophy were combined with those of atypical Pick's disease ${ }^{6}$ and in a large cohort of patients with olivopontocerebellar atrophy. ${ }^{7}$ In this latter study, the inclusion-bearing cells were unequivocally identified as oligodendrocytes by using Leu-7 antibody. Interestingly, although all the inclusions were positive for ubiquitin, some also reacted with an antibody to Rosenthal fibres. Immunoelectron microscopy revealed electron dense particles in relation to both these antibodies to be localised on the constituents of these glial inclusions. The authors have offered two possible explanations for their origin. The inclusions may be formed by normal cytoskeletal structures, most likely by microtubules that undergo degeneration as the epitopes of normal cytoskeletal proteins gradually disappear. The ultrastructural similarities between the tubular constituents of the oligodendroglial 


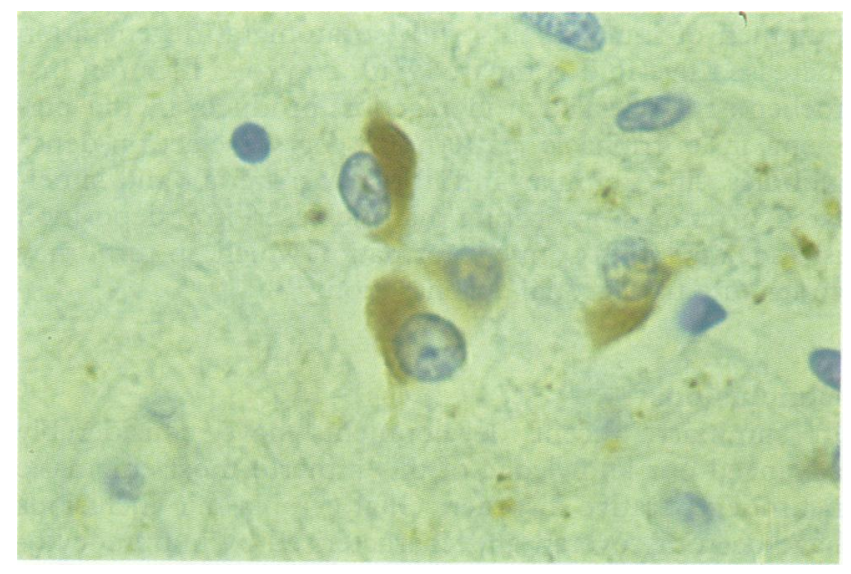

Figure 4 Higher power clearly demonstrates the oligodendroglial localisation of the inclusions in the medulla. Ubiquitin immunocytochemistry, $A B C$ method. Magnification $\times 400$, increased by $240 \%$ for publication.

inclusions and normal microtubules would support this mechanism. Alternatively, portions of the inclusions may consist of abnormal proteins, different from normal cytoskeletal proteins. Although GCIs have been reported in all cases of $\mathrm{MSA},{ }^{89}$ they were absent in a single case of autosomal dominant olivopontocerebellar atrophy, ${ }^{9}$ and in nine cases of the Menzel type, ${ }^{10}$ indicating that this variant does not belong to MSA. GCI has become a diagnostic hallmark of MSA: the occurrence of these inclusions in the olfactory bulb in a clinically unsuspected case of MSA enabled the correct diagnosis to be made. ${ }^{11}$

\section{Other inclusions in MSA}

The original observation ${ }^{1}$ of glial cytoplasmic inclusions was extended by Papp and Lantos ${ }^{12}$ who described the accumulation of abnormal tubular structures in five cellular locations: in oligodendroglial cytoplasm and nuclei, in neuronal cytoplasm and nuclei, and in axons. The neuronal cytoplasmic inclusions were similar to those observed by Kato et $a l^{5}$ and stained only with antibody to ubiquitin. Neuronal cytoplasmic inclusions occurred most frequently in the pons and putamen, but were also seen in the subthalamic nucleus, inferior olivary nucleus, and arcuate nuclei. Although their tinctorial and immunocytochemical profiles were the same, neuronal cytoplasmic inclusions in the pons and putamen were different: pontine inclusions were round, ovoid or reniform with well-demarcated border, whereas putaminal inclusions consisted of an ill-defined, fine network of filamentous structures occupying most of the cytoplasm. Neuronal nuclear inclusions affect the same neuronal populations as the cytoplasmic inclusions, but may also develop in other regions-for example, in the motor cortex. In Gallyas silver-impregnated preparations they appeared as a network of threads and fibrils arranged along or near the nuclear membrane, sometimes filling most of the karyoplasm.

In the same preparations numerous argyrophilic, neuronal processes and threads were also noted, particularly in regions rich in neuronal or glial inclusions. In the basis pontis their course and morphology resembled degenerated axons. Glial cells containing cytoplasmic inclusions sometimes had one or two rod-like inclusions in their nucleus. These oligodendroglial nuclear inclusions are similar to nuclear rods in normal cells. High-resolution electron micrographs revealed tubular structures as the major component of inclusion bodies in all five intracellular locations. The diameter of the tubular structures at their smooth part is within the range of those of GCIs: 20-30 $\mathrm{nm}$. They have side extensions and a fuzzy cover closely associated with them. The side extensions correspond to circular or wheel-shaped profiles with a diameter varying between 50 and $120 \mathrm{~nm}$. These observations indicate that the side extensions are more complex than osmiophilic granules as suggested by some authors. ${ }^{45}$ The nature of these side extensions should be further investigated, as they may provide insight into the formation of inclusions in MSA. It has to be emphasised, however, that high densities of GCIs occur extensively in the brain, whereas neuronal cytoplasmic and neuronal nuclear inclusions develop in a large number only in a small proportion of some neuronal populations. The significance of these findings is twofold.

Firstly, they establish the common cellular pathology of striatonigral degeneration, olivopontocerebellar atrophy and Shy-Drager syndrome and thus provide a diagnostic hallmark in addition to the previously described, non-specific histological features.

Secondly, the occurrence of these inclusions in all three conditions confirms that they are all variants of MSA and defines MSA as a group of diseases with similar pathology or as a nosological entity.

An immunocytochemical and ultrastructural investigation of neuronal cytoplasmic ${ }^{13}$ and oligodendroglial cytoplasmic $^{14}$ inclusions, while confirming previous findings, has also provided new information. Some neuronal cytoplasmic inclusions were stained with antibodies against phosphorylated neurofilaments and most oligodendroglial cytoplasmic inclusions were positive not only for ubiquitin, but also for $a \mathrm{~B}$-crystallin, a lens protein and a major component of Rosenthal fibres. These latter inclusions also immunoreacted, to a varying degree, with antibodies to tubulin, tau protein, and paired helical filaments. The staining with an antibody to $a \mathrm{~B}$-crystallin, however, is not specific, as a positive reaction has been seen in other neurological diseases and, to a lesser extent, in normal controls. This immunocytochemical profile suggests an interaction between ubiquitin, aB-crystallin and cytoskeletal proteins in the formation of these inclusions.

In the white matter, oligodendroglial inclusions also immunostained with antibodies to paired helical filaments and one of the microtubule-associated proteins (MAP), MAP5, in addition to the usual ubiquitin and tau staining. ${ }^{15}$ In the putamen, pontine nuclei and inferior olivary nuclei, neuronal and oligodendroglial cells gave positive reactions with all these antibodies. More surprisingly, in all these sites, astrocytes were positively stained with all four antibodies, with the exception of being tau-negative in the putamen. Compared with silver impregnation according to Gallyas, in this study argyrophilia closely correlated with paired helical filament and tau protein immunoreactivities. ${ }^{15}$ MAP5 positivity of GCI was also convincingly demonstrated in other studies, ${ }^{1016}$ but this antigen was also expressed in other glial cells that contained no GCIs, mainly in reactive microglia and oligodendrocytes. ${ }^{16}$ Phosphorylated highmolecular-weight neurofilament protein was also demonstrated in the ventral horn cells in MSA. ${ }^{17}$

\section{The distribution of inclusions}

The distribution of oligodendroglial inclusions has been recently investigated in a semiquantitative analysis of 
Gallyas silver impregnated preparations of 14 brains and 11 spinal cords of patients with MSA. ${ }^{18}$ The results show a system-bound, extensive degeneration of interfascicular, perineuronal, and perivascular oligodendrocytes. GCI-rich structures in the suprasegmental motor systems (primary motor and higher motor areas of the cerebral cortex, pyramidal, extrapyramidal, and corticocerebellar systems), in the supraspinal autonomic systems, and in their targets. In contrast, the visual, and auditory pathways, olfactory structures, somatosensory systems, association and limbic cortical areas and subcortical limbic structures contain no or only a few GCIs. Comparison of the severity of oligodendroglial degeneration, as demonstrated by GCI density, with that of neuronal alterations (neuronal cytoplasmic and nuclear inclusions, degenerated neuronal processes and loss of nerve cells), indicates a striking preponderance of oligodendroglial degeneration. This in turn, implies that neither degeneration of axons, nor of neuronal cell bodies is a prerequisite of the development of GCIs. These findings suggest that oligodendroglial degeneration may occur before apparent morphological alterations in axons and neuronal cell bodies, and argue against GCIs developing as a reaction against the axonal damage. Moreover, these results show that GCI may cause, or contribute to, the manifestation of clinical symptomatology in structures rich in GCIs, but without significant neuronal alterations. These include cerebral motor cortical areas and the reticular formation of the lower brainstem, both previously thought to be spared in MSA.

\section{Neuronal loss}

The involvement of the cerebral cortex was further demonstrated in olivopontocerebellar atrophy. Laminar astrocytosis was found in the fifth layer of the motor cortex in four out of six brains and the pattern of astrocytosis was different from the distribution of GCIs. The authors considered the change to reflect the pathology in the primary motor cortex. ${ }^{19}$

Another line of investigation which has shed light on the pathogenetic process has been quantitative analysis of neuronal populations. Nerve cell counts in the lateral horn of the spinal cords were carried out in 15 cases of MSA: eight with severe postural hypotension, three with orthostatic hypotension and four without any evidence of autonomic failure. The number of neurons was reduced by half in all cases, but nerve cell loss could not be correlated with the degree of autonomic failure. The authors assumed that pathology in other sites-for example, sympathetic ganglia or the dorsal vagal nuclei, may explain this finding. ${ }^{20}$ Neuronal loss was also assessed in the striatum and substantia nigra in seven cases of MSA. ${ }^{21}$ Depletion of striatal small cells and nigral pigmented cells was more prominent in the caudal part than in the rostral or mid-parts of these structures, cell loss being particularly severe in the dorsolateral zone of the caudal putamen and in the lateral zone of the caudal substantia nigra.

These findings suggest that MSA chiefly affects the striatal and nigral efferent systems which connect the caudal and dorsolateral putamen with the caudolateral nigra. In less severe cases only the caudal parts were affected, indicating that the degenerative process of MSA may start in the caudal parts of the putamen and substantia nigra and extend only later to the rostral portions. The overall pattern, therefore, suggests that striatal small cells and pigmented cells of the substantia nigra degenerate according to anatomical relationship. A further cellular abnormality of MSA is the deposition of granular, iron-containing pigment in the atrophied striatum. Iron content in the putamen of four patients with striatonigral degeneration increased five-fold compared with controls. Electron histochemistry revealed iron reaction products in the pigment in a triphasic pattern of coarse, electron-dense globules, fine granular and fibrillary material, and lamellated structures, indicating that increased iron deposition is associated with pigment formation which, in turn, may contribute to neuronal degeneration. ${ }^{22}$

\section{Conclusions}

In summary, recent developments have considerably advanced our knowledge of this hitherto most enigmatic neurodegenerative disorder. Glial cytoplasmic inclusions have been discovered and characterised by immunocytochemistry and ultrastructure. Inclusions in neuronal cytoplasms and nuclei, in axons, and in oligodendroglial nuclei have been subsequently reported. The distribution pattern of these inclusions and quantitative analysis of neuronal populations have indicated that these abnormalities are not haphazard, but affect particular systems and neuronal populations.

The significance of these developments is manifold. Firstly, the glial cytoplasmic inclusions provide a histological hallmark for MSA, thus increasing diagnostic accuracy. Even if these inclusions were not specific for MSA, ${ }^{23}$ their importance in neuropathological studies matches that of Lewy bodies in Parkinson's disease or senile plaques and neurofibrillary tangles in Alzheimer's disease.

Secondly, since they are consistently and equally found in striatonigral degeneration, olivopontocerebellar atrophy and Shy-Drager syndrome, their presence supports earlier notions that these conditions form a nosological entity or a group of diseases with similar pathology. They also define the term MSA which should be reserved for these conditions.

Thirdly, GCIs highlight the importance of glial pathology in neurodegenerative disease. Although glial, particularly astrocytic, and microglial changes have been known to occur in neurodegeneration, GCIs in MSA are the first glial abnormalities which appear to be the hallmark lesion of a neurodegenerative disease. Moreover, recent evidence suggests that they may precede neuronal alteration and are unlikely to represent a reaction to axonal injury.

Fourthly, the pathology of MSA is far more extensive than hitherto considered and involves the cerebral cortex. This realisation should contribute to the understanding of some of the clinical symptomatology.

Finally, the distribution of cellular abnormalities and neuronal loss is not haphazard, but involves particular systems and neuronal populations.

Further studies are needed to establish the molecular pathology of GCI in order to understand fully the pathogenesis of MSA. The absence of GCIs in familial forms of olivopontocerebellar atrophy is of considerable interest, as it suggests a different pathogenetic mechanism. Molecular genetics may help to elucidate the cause of this phenotypic difference. Moreover, immunochemical investigations of the GCI could not only throw light on the composition of these inclusions in a relatively rare neurological disease, but may also give further insight into the mechanism of cytoskeletal abnormalities in other, more common neurodegenerative disorders.

Department of Neuropathology,

P L LANTOS

Institute of Psychiatry,

London SE5 8AF, UK

Department of Neurology,

M I PAPP

Semmelweis University Medical School,

H-1083 Budapest, Hungary 
1 Papp MI, Kahn JE, Lantos PL. Glial cytoplasmic inclusions in the CNS of patients with multiple system atrophy (striatonigral degeneration, of patients with multiple system atrophy (striatonigral degeneration, olivopontocerebella

2 Gallyas F. Silver staining of Alzheimer's neurofibrillary changes by means of physical development. Acta Morphol Acad Sci Hung 1971;19:1-8.

3 Gallyas F, Wolff JR. Metal-catalyzed oxidation renders silver intensification selective. Application for the histochemistry of diaminobenzidine and neurofibrillary changes. F Histochem Cytochem 1986;34:1667-72.

4 Nakazato $Y$, Yamazaki $H$, Hirato J, Ishida Y, Yamaguchi $H$. Oligodendroglial microtubular tangles in olivopontocerebellar atrophy. f Neuropathol Exp Neurol 1990;49:521-30.

5 Kato S, Nakamura H. Cytoplasmic argyrophilic inclusions in neurons of pontine nuclei in patients with olivopontocerebellar atrophy: immunopontine nuclei in patients with olivopontocerebellar atrophy: immuno79:584-94.

6 Horoupian DS, Dickson DW. Striatonigral degeneration, olivopontocerebellar atrophy and "atypical" Pick disease. Acta Neuropathol 1991;

7 Kato S, Nakamura H, Hirano A, Ito H, Llena JF, Yen S-H. Argyrophilic ubiquitinated cytoplasmic inclusions of Leu-7-positive glial cells in olivopontocerebellar atrophy (multiple system atrophy). Acta Neuropathol 1991;82:488-93.

8 Mochizuki A, Mizusawa H, Ohkoshi N, et al. Argentophilic intracytoplasmic inclusions in multiple system atrophy. F Neurol 1992;239: 311-6.

9 Costa C, Duykaerts C, Cervera P, Hauw J-J. Les inclusions oligodendrogliales, un marqueur des atrophies multisystématisées. Rev Neurol (Paris) 1992;4:274-80.

10 Abe $\mathrm{H}$, Yagishita $\mathrm{S}$, Amano $\mathrm{N}$, Iwabuchi $\mathrm{K}$, Hasegawa $\mathrm{K}$, Kowa $\mathrm{K}$. Argyrophilic glial intracytoplasmic inclusions in multiple system atrophy: immunocytochemical and ultrastructural study. Acta Neuropathol phy: immunocytc

11 Daniel SE, Hawkes CH. Preliminary diagnosis of Parkinson's disease by olfactory bulb pathology. Lancet 1992;340:186.

12 Papp MI, Lantos PL. Accumulation of tubular structures in oligodendroglial and neuronal cells as the basic alteration in multiple system atrophy. $\mathcal{F}$ Neurol Sci 1992;107:172-82.
13 Arima K, Murayama S, Mukoyama M, Inose T. Immunocytochemical and ultrastructural studies of neuronal and oligodendroglial cytoplasmic inclusions in multiple system atrophy. 1. Neuronal cytoplasmic inclusions. Acta Neuropathol 1992;83:453-60

14 Murayama S, Arima K, Nakazato Y, Satoh J-I, Oda M, Inose T. Immunocytochemical and ultrastructural studies of neuronal and oligodendroglial cytoplasmic inclusions in multiple system atrophy. 2 Oligodendroglial cytoplasmic inclusions. Acta Neuropathol 1992;84: 32-8.

15 Kobayashi K, Miyazu K, Katsukawa K, et al. Cytoskeletal protein abnormalities in patients with olivopontocerebellar atrophy - an immunocytochemical and Gallyas silver impregnation study. Neuropathol Appl Neurobiol 1992;18:237-49.

16 Arai $N$, Nishimura $M$, Oda $M$, Morimatsu $Y$, Ohe $R$, Nagatomo $H$. Immunohistochemical expression of microtubule-associated protein 5 (MAP5) in glial cells in multiple system atrophy. $\mathcal{f}$ Neurol Sci 1992;109:102-6.

17 Itoh T, Sobue G, Ken E, Mitsuma T, Takahashi A, Trojanowski JQ Phosphorylated high molecular weight neurofilament protein in the peripheral motor, sensory and sympathetic neuronal perikarya: systemdependent normal variations and changes in amyotrophic lateral sclerosis and multiple system atrophy. Acta Neuropathol 1992;83:240-5.

18 Papp MI, Lantos PL. The distribution of oligodendroglial inclusions in multiple system atrophy and its relevance to clinical symptomatology. Brain 1994;117 (in press)

19 Fujita T, Doi M, Ogata T, Kanazawa I, Mizusawa H. Cerebral cortical pathology of sporadic olivopontocerebellar atrophy. $f$ Neurol Sci 1993;116:41-6.

20 Gray F, Vincent D, Hauw JJ. Quantitative study of lateral horn cells in 15 cases of multiple system atrophy. Acta Neuropathol 1988;75:513-8.

21 Kume A, Takahashi A, Hashizume Y. Neuronal cell loss of the striatonigral system in multiple system atrophy. $\mathcal{f}$ Neurol Sci 1993;117:33-40.

22 Kato S, Meshitsuka S, Ohama E, Tanaka J, Llena JF, Hirano A. Increased iron content in the putamen of patients with striatonigral degeneration. Acta Neuropathol 1992:84:328-30.

23 Yamada T, McGeer PL. Oligodendroglial microtubular masses: an abnormality observed in some human neurodegenerative diseases. Neurosc Lett 1990;120:163-6.

\section{Neurological stamp}

\section{Dominique Jean Larrey (1766-1842)}

Larrey, chief surgeon of Napoleon's grand army, added new dimension to military medicine, in particular, sanitation, epidemiology, procurement of food and supplies for the sick and wounded, training of medical personnel, transport of the injured, and provision for definitive care at the front. He took part in 60 battles and 400 engagements. Larrey instituted immediate care on the battlefield and "flying ambulances" for the rapid evacuation of the wounded from the field of action. Incredible numbers of wounded passed through his hands, affording him enormous clinical experience. An ardent advocate of early amputation he performed as many as 200 in a single day. Large numbers of disarticulations at the shoulder joint and even at the hip joint, with a high incidence of recovery were performed at the front or on the battlefield without anaesthesia. Other major operative procedures included trephining, thoracotomy, and the treatment of penetrating wounds to the abdomen.

In his Memoir on traumatic tetanus, amputation of the limb at the first appearance of the symptoms was advised. $\mathrm{He}$ cites numerous reports of patients salvaged by this operation.

This French postage stamp (Stanley Gibbons 1666, Scott B386) was issued in 1964. His memoirs, modelled after those of Ambrose Paré, provide a fascinating record of the campaigns, medical problems encountered, and the scenes and customs of the many lands traversed by the grand army.

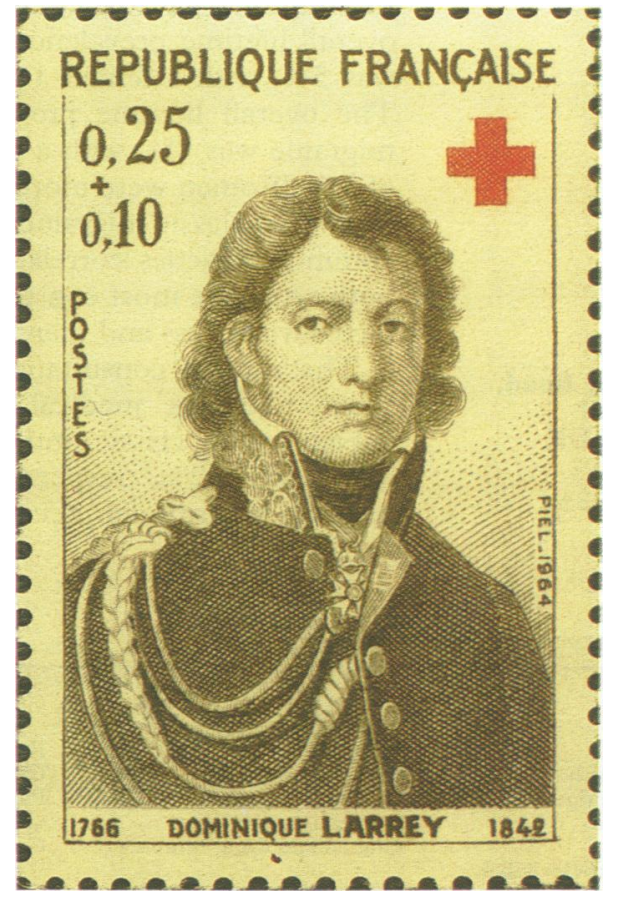

